\title{
Statistical inference for doubly geometric process with exponential distribution
}

\author{
Mustafa Hilmi Pekalp*1 (D), Gültaç Eroğlu İnan² (D), Halil Aydoğdu² (D) \\ ${ }^{1}$ Department of Actuarial Sciences, Faculty of Applied Sciences, Ankara University, Ankara, Turkey \\ ${ }^{2}$ Department of Statistics, Faculty of Science, Ankara University, Ankara, Turkey
}

\begin{abstract}
The geometric process is widely applied as a stochastic monotone model in many practical applications since its introduction. However, it sometimes does not satisfy some requirements in the real-world applications due to model limitations. For this reason, it is proposed a new stochastic model which is called doubly geometric process. In the applications of the doubly geometric process, the estimation problem associated with the process arises naturally. In this study, the statistical inference problem for the doubly geometric process is considered by assuming that the distribution of the first interarrival time has an exponential distribution. The maximum likelihood method is used to estimate the model parameters of the doubly geometric process and the parameter of distribution. The joint distribution of the maximum likelihood estimators is obtained. A simulation study is presented to evaluate the small sample performance of the estimators with different parameter values. Finally, three real-world-data sets are used to illustrate the applicability of the methods.
\end{abstract}

Mathematics Subject Classification (2020). 60K99, 62M99, 62F12

Keywords. Geometric process, doubly geometric process, maximum likelihood estimation, asymptotic normality, exponential distribution

\section{Introduction}

The geometric process (GP) has proved to be a powerful tool in various field of statistics and applied probability since its introduction. Many authors make a significant effort on the GP and a substantial amount of research are published. For example, the GP is used as a model in modelling of an epidemic disease [4], software reliability [9,11], maintenance [15], warranty analysis [2,8], and electricity prices [5]. The definition of this process is given as follows.

Let $\{N(t), t \geq 0\}$ be a counting process $(\mathrm{CP})$ and $\left\{X_{k}, k=1,2, \ldots\right\}$ be the sequence of nonnegative random variables representing the inter-arrival times of this process. The CP $\{N(t), t \geq 0\}$ is said to be a GP with the ratio $a$ if there exists a real number $a>0$ such

\footnotetext{
*Corresponding Author.

Email addresses: mpekalp@ankara.edu.tr (M.H. Pekalp), geroglu@ankara.edu.tr (G. Eroğlu İnan), aydogdu@ankara.edu.tr (H. Aydoğdu)

Received: 22.08.2020; Accepted: 02.07.2021
} 
that $a^{k-1} X_{k}$ for $k=1,2, \ldots$ generate a renewal process (RP) with a common distribution function $F$.

Let $\{N(t), t \geq 0\}$ be a GP with the ratio parameter $a$ and $F_{k}$ be the distribution function of $X_{k}, k=1,2, \ldots$. The distribution function of the first interarrival time of a GP uniquely determines the distribution function $F_{k}$, that is, $F_{k}(x)=F\left(a^{k-1} x\right)$ for $k=1,2, \ldots$. The sequence $\left\{X_{k}, k=1,2, \ldots\right\}$ is stochastically increasing if $a<1$ and stochastically decreasing if $a>1$, respectively. When $a=1$, the GP reduces to a RP.

Despite the GP is widely used as a stochastic monotone model in many practical applications, it has some limitations which are given as follows: (1) If the distributions of the interarrival times have varying shape parameters, the GP will not be used to model these interarrival times. To elaborate, let us take the Weibull distribution as a distribution of the first interarrival time of the GP. It is obvious that the distribution of $X_{1}$ is $F(x)=1-e^{-\left(\frac{x}{\beta}\right)^{\alpha}}, x>0 ; \alpha, \beta>0$. From the definition of the GP, the distribution function of $X_{k}$ is $F_{k}(x)=F\left(a^{k-1} x\right)=1-e^{-\left(\frac{a^{k-1} x}{\beta}\right)^{\alpha}}, k=1,2, \ldots$ As can be seen, the scale parameter $\frac{a^{k-1}}{\beta}$ depends on $k$ and changes over $k$ 's. However, the shape parameter $\alpha$ remains constant over $k$ 's since it does not include $k$. This may restrict the usage of the GP in wider applications. (2) The GP can only be used for stochastically monotone interarrival times. However, in real world applications, the pattern of the interarrival times can be non-monotonous. In such cases, the GP will not be the suitable model.

Due to these limitations of the GP, there is a need to propose new models in the real-world applications. In recent years, many authors have made a significant effort on developing new methods to model the data from a series of events, see [12-14] for the details. The current paper focuses on the doubly geometric process (DGP) proposed by [12] which can overcome the limitations mentioned above. This process is defined in the following way.

The CP $\{N(t), t \geq 0\}$ is said to be a DGP with the parameter $a$ if there exists a real number $a>0$ such that $a^{k-1} X_{k}^{h(k)}, k=1,2, \ldots$ generate a RP with a common distribution function $F$ where $h(k)>0$ is a function of $k$ with $h(1)=1$ for $k=1,2, \ldots$ [12].

Since $\mathrm{Wu}[12]$ obtains that the DGP with $h(k)=(1+\log (k))^{b}$ outperforms the processes with the other $h(k)$ 's which are $b^{k-1}, b^{\log (k)}$, and $1+b \log (k), h(k)$ is taken as $h(k)=$ $(1+\log (k))^{b}$ where $b$ is a real number and $\log$ is the logarithm with base 10 .

Let $\{N(t), t \geq 0\}$ be a DGP with the parameters $a$ and $b$, and $F_{k}$ be the distribution function of $\left\{X_{k}, k=1,2, \ldots\right\}$, then, it is obvious from the definition of the DGP that $F_{k}(x)=F\left(a^{k-1} x^{(1+\log (k))^{b}}\right)$ for $k=1,2, \ldots$.

Monotonicity property of the DGP can be given as follows: i) If $0<a<1, b<0$, and $P\left(X_{1}>1\right)=1$ or if $0<a<1,0<b<4.898226$, and $P\left(0<X_{1}<1\right)=1$, then $\left\{X_{k}, k=1,2, \ldots\right\}$ is stochastically increasing. ii) If $a>1, b<0$, and $P\left(0<X_{1}<1\right)=1$ or if $a>1,0<b<4.898226$, and $P\left(X_{1}>1\right)=1$, then $\left\{X_{k}, k=1,2, \ldots\right\}$ is stochastically decreasing iii) If $(1+\log (k+1))^{-b}(\log (y)-k \log (a))+(1+\log (k))^{-b}((k-1) \log (a)-\log (y))$ varies between negative and positive values, then $\left\{X_{k}, k=1,2, \ldots\right\}$ is non-monotonous over $k$ 's, where $y$ represents all possible values on $X_{k}, k=1,2, \ldots$ [12].

In the applications of the DGP, the estimation problem of model parameters naturally arises. In this study, under the assumption that the first interarrival time follows an exponential distribution, we consider the estimation problem of the model parameters of the DGP. The remainder of this paper is structured as follows: In Section 2, the maximum likelihood (ML) method is used to obtain the estimators of the model parameters. The asymptotic joint distribution of the ML estimators is given. In Section 3, a simulation study is performed to evaluate the small sample performance of the estimators with different parameter values by using the bias and mean square error (MSE) criteria. To illustrate the applicability of the methods given in previous sections, three real world applications, 
namely the hydraulic system failure data (Data set 1), USS Halfbeak No. 3 propulsion diesel engine failure data (Data set 2), and the Aircraft 3 data (Data set 3), are considered. Finally, we present the conclusion part of the study.

\section{Statistical inference for the DGP}

The exponential distribution is widely used as lifetime distribution in the reliability context and life testing areas. Its probability density function (pdf) is given by

$$
f(x)=\frac{1}{\theta} e^{-\frac{x}{\theta}}, x>0 ; \theta>0 .
$$

Note that the expected value and the variance of exponential distribution are $\theta$ and $\theta^{2}$, respectively. Assume that $\left\{X_{1}, X_{2}, \ldots, X_{n}\right\}$ is a data set which comes from a DGP with ratio parameter $a$ and $h(k)=(1+\log (k))^{b}$. $X_{1}$ is distributed as exponential distribution with mean $\theta$. Then, based on this data set, the log-likelihood function can be obtained as

$$
\begin{aligned}
\ln L(a, b, \theta)=\frac{n(n-1)}{2} \ln a & -n \ln \theta-\frac{1}{\theta}\left(\sum_{k=1}^{n} a^{k-1} x_{k}^{(1+\log (k))^{b}}\right) \\
& +\sum_{k=1}^{n}\left((1+\log (k))^{b}-1\right) \ln \left(x_{k}\right)+\sum_{k=1}^{n} \ln (1+\log (k))^{b} .
\end{aligned}
$$

By differentiating the log-likelihood function with respect to $a, b$, and $\theta$, and equating them to zero, the likelihood equations are

$$
\begin{aligned}
\frac{\partial \ln L}{\partial a}= & \frac{n(n-1)}{2}-\frac{1}{\theta} \sum_{k=1}^{n}(k-1) a^{k-2} x_{k}^{(1+\log (k))^{b}}=0 \\
\frac{\partial \ln L}{\partial b}= & -\frac{1}{\theta}\left(\sum_{k=1}^{n} a^{k-1} x_{k}^{(1+\log (k))^{b}} \ln x_{k}(1+\log (k))^{b} \ln (1+\log (k))\right) \\
& +\sum_{k=1}^{n} \ln x_{k}(1+\log (k))^{b} \ln (1+\log (k))+\sum_{k=1}^{n} \ln (1+\log (k))=0,
\end{aligned}
$$

and

$$
\frac{\partial \ln L}{\partial \theta}=-\frac{n}{\theta}+\frac{1}{\theta^{2}} \sum_{k=1}^{n} a^{k-1} x_{k}^{(1+\log (k))^{b}}=0 .
$$

The ML estimators of the parameters $a, b$, and $\theta$ are obtained by solving the likelihood equations given above. However, the ML estimators cannot be obtained explicitly. Any package programme, for example Mathematica, can be used to solve these equations simultaneously to obtain the ML estimators of the model parameters $a, b$, and $\theta$. On the other hand, the log-likelihood function given in Equation 2.1 can be maximized directly to obtain the ML estimators of the model parameters $a, b$, and $\theta$.

By [3], the joint distribution of the ML estimators is an asymptotically normal as $n \rightarrow \infty$ with mean vector $(a, b, \theta)$ and variance-covariance matrix $I^{-1}$ as follows:

$$
\left(\begin{array}{l}
\hat{a} \\
\hat{b} \\
\hat{\theta}
\end{array}\right) \sim A N\left(\left(\begin{array}{l}
a \\
b \\
\theta
\end{array}\right), I^{-1}\right)
$$


where $I^{-1}$ is the inverse of the Fisher information matrix $I$. Here, $I^{-1}$ is given as follows:

$$
I^{-1}=\left[\begin{array}{lll}
u_{11} & u_{12} & u_{13} \\
& u_{22} & u_{23} \\
& & u_{33}
\end{array}\right]
$$

where

$$
\begin{aligned}
& u_{11}=-12 a^{2}\left(\left(\sum_{k=1}^{n}\left(0.422784-\ln \left(a^{k-1}\right)+\ln (\theta) \ln (1+\log (k))\right)^{2}-n \sum_{k=1}^{n}\left(1+\ln \left(a^{k-1}\right)^{2}\right.\right.\right. \\
& \left.-2 \ln \left(a^{k-1}\right)(0.422784+\ln (\theta))+0.16667(4.94208+6 \ln (\theta)(0.845569+\ln (\theta)))\right) \\
& \left(\ln (1+\log (k))^{2}\right) /\left(n\left(\left(-2+6 n-4 n^{2}\right) \sum_{k=1}^{n}\left(0.422784-\ln \left(a^{k-1}\right)+\ln (\theta)\right) \ln (1+\log (k))\right)^{2}\right. \\
& +12(n-1)\left(\sum_{k=1}^{n}\left(0.422784-\ln \left(a^{k-1}\right)+\ln (\theta)\right) \ln (1+\log (k))\right) \sum_{k=1}^{n}(k-1)\left(0.422784-\ln \left(a^{k-1}\right)\right. \\
& +\ln (\theta)) \ln (1+\log (k))-12\left(\sum_{k=1}^{n}(k-1)\left(0.422784-\ln \left(a^{k-1}\right)+\ln (\theta)\right) \ln (1+\log (k))\right)^{2} \\
& +n\left(n^{2}-1\right) \sum_{k=1}^{n}\left(1+\ln \left(a^{k-1}\right)^{2}-2 \ln \left(a^{k-1}\right)(0.422784+\ln (\theta))+0.16667(4.94208+6 \ln (\theta)\right. \\
& \left.\left.(0.845569+\ln (\theta)))) \ln (1+\log (k))^{2}\right)\right), \\
& u_{12}=\left(6 a(n-1) \sum_{k=1}^{n}\left(0.422784-\ln \left(a^{k-1}\right)+\ln (\theta)\right) \ln (1+\log (k))-2 \sum_{k=1}^{n}(k-1)\left(0.422784-\ln \left(a^{k-1}\right)\right.\right. \\
& +\ln (\theta)) \ln (1+\log (k))) /\left(\left(-2+6 n-4 n^{2}\right)\left(\sum_{k=1}^{n}\left(0.422784-\ln \left(a^{k-1}\right)+\ln (\theta)\right) \ln (1+\log (k))\right)^{2}\right. \\
& \left.+12(n-1)\left(\sum_{k=1}^{n}\left(0.422784-\ln \left(a^{k-1}\right)+\ln \theta\right)\right) \ln (1+\log (k))\right) \sum_{k=1}^{n}(k-1)\left(0.422784-\ln \left(a^{k-1}\right)\right. \\
& +\ln (\theta)) \ln (1+\log (k))-12\left(\sum_{k=1}^{n}(k-1)\left(0.422784-\ln \left(a^{k-1}\right)+\ln (\theta)\right) \ln (1+\log (k))\right)^{2} \\
& +n\left(n^{2}-1\right) \sum_{k=1}^{n}\left(1+\ln \left(a^{k-1}\right)^{2}-2 \ln \left(a^{k-1}\right)(0.422784+\ln (\theta))+0.16667(4.94208+6 \ln (\theta)\right. \\
& \left.(0.845569+\ln (\theta)))) \ln (1+\log (k))^{2}\right), \\
& u_{13}=-6 a \theta\left(2\left(\sum_{k=1}^{n}\left(0.422784-\ln \left(a^{k-1}\right)+\ln (\theta)\right) \ln (1+\log (k))\right) \sum_{k=1}^{n}(k-1)\left(0.422784-\ln \left(a^{k-1}\right)+\ln (\theta)\right)\right. \\
& \ln (1+\log (k))-n(n-1) \sum_{k=1}^{n}\left(1+\ln \left(a^{k-1}\right)^{2}-2 \ln \left(a^{k-1}\right)(0.422784+\ln (\theta))+0.16667(4.94208+\right. \\
& \left.6 \ln (\theta)(0.845569+\ln (\theta)))) \ln (1+\log (k))^{2}\right) /\left(n \left(( - 2 + 6 n - 4 n ^ { 2 } ) \left(\sum_{k=1}^{n}\left(0.422784-\ln \left(a^{k-1}\right)+\ln (\theta)\right)\right.\right.\right. \\
& \ln (1+\log (k)))^{2}+12(n-1)\left(\sum_{k=1}^{n}\left(0.422784-\ln \left(a^{k-1}\right)+\ln (\theta)\right) \ln (1+\log (k))\right) \sum_{k=1}^{n}(k-1)(0.422784 \\
& \left.-\ln \left(a^{k-1}\right)+\ln (\theta)\right) \ln (1+\log (k))-12\left(\sum_{k=1}^{n}(k-1)\left(0.422784-\ln \left(a^{k-1}\right)+\ln (\theta)\right) \ln (1+\log (k))\right)^{2} \\
& +n\left(n^{2}-1\right) \sum_{k=1}^{n}\left(1+\ln \left(a^{k-1}\right)^{2}-2 \ln \left(a^{k-1}\right)(0.422784+\ln (\theta))+0.16667(4.94208+6 \ln (\theta)\right. \\
& \left.\left.(0.845569+\ln (\theta)))) \ln (1+\log (k))^{2}\right)\right),
\end{aligned}
$$




$$
\begin{aligned}
u_{22} & =\left(n\left(n^{2}-1\right)\right) /\left(\left(-2+6 n-4 n^{2}\right)\left(\sum_{k=1}^{n}\left(0.422784-\ln \left(a^{k-1}\right)+\ln (\theta)\right) \ln (1+\log (k))\right)^{2}+12(n-1)\right. \\
& \left(\sum_{k=1}^{n}\left(0.422784-\ln \left(a^{k-1}\right)+\ln (\theta)\right) \ln (1+\log (k))\right) \sum_{k=1}^{n}(k-1)\left(0.422784-\ln \left(a^{k-1}\right)+\ln (\theta)\right) \\
& \ln (1+\log (k))-12\left(\sum_{k=1}^{n}(k-1)\left(0.422784-\ln \left(a^{k-1}\right)+\ln (\theta)\right) \ln (1+\log (k))\right)^{2}+n\left(n^{2}-1\right) \\
& \sum_{k=1}^{n}\left(1+\ln \left(a^{k-1}\right)^{2}-2 \ln \left(a^{k-1}\right)(0.422784+\ln (\theta))+0.16667(4.94208+6 \ln (\theta)(0.845569+\right. \\
& \left.\ln (\theta)))) \ln (1+\log (k))^{2}\right), \\
u_{23} & =\left(2 ( n - 1 ) \theta \left((2 n-1) \sum_{k=1}^{n}\left(0.422784-\ln \left(a^{k-1}\right)+\ln (\theta)\right) \ln (1+\log (k))-3 \sum_{k=1}^{n}(k-1)(0.422784\right.\right. \\
& \left.\left.\left.-\ln \left(a^{k-1}\right)+\ln (\theta)\right) \ln (1+\log (k))\right)\right) /\left(( - 2 + 6 n - 4 n ^ { 2 } ) \left(\sum_{k=1}^{n}\left(0.422784-\ln \left(a^{k-1}\right)+\ln (\theta)\right)\right.\right. \\
& \ln (1+\log (k)))^{2}+12(n-1)\left(\sum_{k=1}^{n}\left(0.422784-\ln \left(a^{k-1}\right)+\ln (\theta)\right) \ln (1+\log (k))\right) \sum_{k=1}^{n}(k-1)(0.422784- \\
& \left.\ln \left(a^{k-1}\right)+\ln (\theta)\right) \ln (1+\log (k))-12\left(\sum_{k=1}^{n}(k-1)\left(0.422784-\ln \left(a^{k-1}\right)+\ln (\theta)\right) \ln (1+\log (k))\right)^{2} \\
& +n\left(n^{2}-1\right) \sum_{k=1}^{n}\left(1+\ln \left(a^{k-1}\right)^{2}-2 \ln \left(a^{k-1}\right)(0.422784+\ln (\theta))+0.16667(4.94208+6 \ln (\theta)(0.845569+\right. \\
& \left.\ln (\theta)))) \ln (1+\log (k))^{2}\right)
\end{aligned}
$$

and

$$
\begin{aligned}
u_{33}= & \left(2 \theta ^ { 2 } \left(-6\left(\sum_{k=1}^{n}(k-1)\left(0.422784-\ln \left(a^{k-1}\right)+\ln (\theta)\right) \ln (1+\log (k))\right)^{2}+n\left(1-3 n+2 n^{2}\right) \sum_{k=1}^{n}(1+\right.\right. \\
& \left.\ln \left(a^{k-1}\right)^{2}-2 \ln \left(a^{k-1}\right)(0.422784+\ln (\theta))+0.16667(4.94208+6 \ln (\theta)(0.845569+\ln (\theta)))\right) \\
& \left.\left.\ln (1+\log (k))^{2}\right)\right) /\left(n \left(\left(-2+6 n-4 n^{2}\right)\left(\sum_{k=1}^{n}\left(0.422784-\ln \left(a^{k-1}\right)+\ln (\theta)\right) \ln (1+\log (k))\right)^{2}+12(n-1)\right.\right. \\
& \left(\sum_{k=1}^{n}\left(0.422784-\ln \left(a^{k-1}\right)+\ln (\theta)\right) \ln (1+\log (k))\right) \sum_{k=1}^{n}(k-1)\left(0.422784-\ln \left(a^{k-1}\right)+\ln (\theta)\right) \\
& \ln (1+\log (k))-12\left(\sum_{k=1}^{n}(k-1)\left(0.422784-\ln \left(a^{k-1}\right)+\ln (\theta)\right) \ln (1+\log (k))\right)^{2}+n\left(n^{2}-1\right) \sum_{k=1}^{n}(1+ \\
& \left.\ln \left(a^{k-1}\right)^{2}-2 \ln \left(a^{k-1}\right)(0.422784+\ln (\theta))+0.16667(4.94208+6 \ln (\theta)(0.845569+\ln (\theta)))\right) \\
& \left.\left.\ln (1+\log (k))^{2}\right)\right) .
\end{aligned}
$$

We give the derivation of $I^{-1}$ in Appendix.

It can be concluded from the Equation 2.2 that the asymptotic distribution of each parameter is

$$
\begin{aligned}
& \hat{a} \sim A N\left(a, u_{11}\right), \\
& \hat{b} \sim A N\left(b, u_{22}\right), \\
& \hat{\theta} \sim A N\left(\theta, u_{33}\right) .
\end{aligned}
$$

It is obvious that the estimators $\hat{a}, \hat{b}$ and $\hat{\theta}$ are asymptotically unbiased and consistent. 


\section{Simulation study}

In this section, a simulation study is performed to evaluate the performance of the estimators of the parameters $a, b$, and $\theta$ by using the bias and MSE criteria. Here, values of the parameter a are chosen as $0.95,0.99,1.01$ and 1.05 since the trend is usually small in practice [7]. To see the effects of the stochastic monotonic property of the DGP on estimators of the parameters, the parameter $b$ is chosen as $2,-2,0.5$, and -0.5 . The mean $\theta$ of the exponential distribution is taken as 2 and 0.5 . It can be observed a realization $\left\{X_{1}, X_{2}, \ldots, X_{n}\right\}$ of a DGP as follows. First, generate a random sample $\left\{Y_{1}, Y_{2}, \ldots, Y_{n}\right\}$ from exponential distribution with mean $\theta$. Then, the transformation

$$
X_{k}=\left(\frac{Y_{k}}{a^{k-1}}\right)^{\frac{1}{(1+\log (k))^{b}}}, k=1,2, \ldots, n
$$

is applied to obtain the data set $\left\{X_{1}, X_{2}, \ldots, X_{n}\right\}$. Based on this data set, we calculate the simulated means, biases and MSEs of the parameters $a, b$, and $\theta$. Throughout the simulation study, we consider the sample sizes $n=30,50$, and 100 and take the repetition number as 1000. The simulated means, biases and MSEs of all estimators are given in the following Tables 1-8.

Table 1. The simulated means, biases and MSE's for the estimators of the parameters $a, b=2$ and $\theta=2$.

\begin{tabular}{|c|c|c|c|c|c|c|c|c|c|c|}
\hline \multirow[b]{2}{*}{$a$} & \multirow[b]{2}{*}{$n$} & \multicolumn{3}{|c|}{$\hat{a}$} & \multicolumn{3}{|c|}{$\hat{b}$} & \multicolumn{3}{|c|}{$\theta$} \\
\hline & & Mean & Bias & MSE & Mean & Bias & MSE & Mean & Bias & MSE \\
\hline \multirow{3}{*}{0.95} & 30 & 0.9442 & -0.0058 & 0.0007 & 2.0741 & 0.0741 & 0.0467 & 2.1109 & 0.1109 & 0.6534 \\
\hline & 50 & 0.9467 & -0.0034 & 0.0002 & 2.0415 & 0.0415 & 0.0203 & 2.0773 & 0.0773 & 0.4082 \\
\hline & 100 & 0.9487 & -0.0014 & 0.0000 & 2.0170 & 0.0170 & 0.0074 & 2.0263 & 0.0263 & 0.1577 \\
\hline \multirow{3}{*}{0.99} & 30 & 0.9876 & -0.0024 & 0.0006 & 2.0782 & 0.0782 & 0.0478 & 2.1750 & 0.1750 & 0.7563 \\
\hline & 50 & 0.9889 & -0.0012 & 0.0001 & 2.0404 & 0.0404 & 0.0205 & 2.1029 & 0.1029 & 0.4355 \\
\hline & 100 & 0.9897 & -0.0003 & 0.0000 & 2.0197 & 0.0197 & 0.0075 & 2.0591 & 0.0591 & 0.1833 \\
\hline \multirow{3}{*}{1.01} & 30 & 1.0088 & -0.0012 & 0.0005 & 2.0706 & 0.0706 & 0.0449 & 2.1239 & 0.1239 & 0.6669 \\
\hline & 50 & 1.0096 & -0.0004 & 0.0001 & 2.0425 & 0.0425 & 0.0216 & 2.0846 & 0.0846 & 0.4385 \\
\hline & 100 & 1.0103 & 0.0003 & 0.0000 & 2.0183 & 0.0183 & 0.0074 & 2.0622 & 0.0622 & 0.2087 \\
\hline \multirow{3}{*}{1.05} & 30 & 1.0520 & 0.0020 & 0.0006 & 2.0819 & 0.0819 & 0.0462 & 2.1582 & 0.1582 & 0.8438 \\
\hline & 50 & 1.0519 & 0.0019 & 0.0002 & 2.0378 & 0.0378 & 0.0203 & 2.1247 & 0.1247 & 0.4461 \\
\hline & 100 & 1.0511 & 0.0011 & 0.0000 & 2.0144 & 0.0144 & 0.0068 & 2.0703 & 0.0703 & 0.2017 \\
\hline
\end{tabular}

Table 2. The simulated means, biases and MSE's for the estimators of the parameters $a, b=-2$ and $\theta=2$.

\begin{tabular}{|c|c|c|c|c|c|c|c|c|c|c|}
\hline \multirow{2}{*}{$\boldsymbol{a}$} & \multirow{n}{*}{$\boldsymbol{n}$} & \multicolumn{3}{|c|}{$\hat{\boldsymbol{a}}$} & \multicolumn{3}{|c|}{$\hat{\boldsymbol{b}}$} & \multicolumn{3}{c|}{$\hat{\boldsymbol{\theta}}$} \\
\cline { 2 - 11 } & $\mathbf{M e a n}$ & Bias & MSE & Mean & Bias & MSE & Mean & Bias & MSE \\
\hline \multirow{3}{*}{$\mathbf{0 . 9 5}$} & $\mathbf{3 0}$ & 0.9447 & -0.0053 & 0.0007 & -1.9226 & 0.0774 & 0.0463 & 2.1767 & 0.1767 & 0.8020 \\
\cline { 2 - 11 } & $\mathbf{5 0}$ & 0.9473 & -0.0023 & 0.0002 & -1.9633 & 0.0368 & 0.0208 & 2.0655 & 0.0655 & 0.3970 \\
\cline { 2 - 11 } & $\mathbf{1 0 0}$ & 0.9489 & -0.0011 & 0.0000 & -1.9843 & 0.0157 & 0.0072 & 2.0450 & 0.0450 & 0.1772 \\
\hline \multirow{3}{*}{$\mathbf{0 . 9 9}$} & $\mathbf{3 0}$ & 0.9878 & -0.0022 & 0.0005 & -1.9286 & 0.0715 & 0.0463 & 2.1530 & 0.1530 & 0.6870 \\
\cline { 2 - 11 } & $\mathbf{5 0}$ & 0.9885 & -0.0015 & 0.0001 & -1.9538 & 0.0462 & 0.0203 & 2.0998 & 0.0998 & 0.4620 \\
\cline { 2 - 10 } & $\mathbf{1 0 0}$ & 0.9896 & -0.0004 & 0.0000 & -1.9785 & 0.0215 & 0.0071 & 2.0434 & 0.0434 & 0.1887 \\
\hline \multirow{3}{*}{$\mathbf{1 . 0 1}$} & $\mathbf{3 0}$ & 1.0087 & -0.0013 & 0.0010 & -1.9289 & 0.0712 & 0.0606 & 2.1861 & 0.1861 & 0.8032 \\
\cline { 2 - 11 } & $\mathbf{5 0}$ & 1.0105 & 0.0005 & 0.0001 & -1.9637 & 0.0363 & 0.0195 & 2.1227 & 0.1227 & 0.4339 \\
\cline { 2 - 10 } & $\mathbf{1 0 0}$ & 1.0101 & 0.0001 & 0.0000 & -1.9878 & 0.0122 & 0.0072 & 2.0336 & 0.0336 & 0.1823 \\
\hline \multirow{3}{*}{$\mathbf{1 . 0 5}$} & $\mathbf{3 0}$ & 1.0525 & 0.0025 & 0.0006 & -1.9238 & 0.0763 & 0.0477 & 2.1652 & 0.1652 & 0.7875 \\
\cline { 2 - 10 } & $\mathbf{5 0}$ & 1.0516 & 0.0016 & 0.0002 & -1.9622 & 0.0378 & 0.0198 & 2.1244 & 0.1244 & 0.4104 \\
\cline { 2 - 10 } & $\mathbf{1 0 0}$ & 1.0510 & 0.0010 & 0.0000 & -1.9852 & 0.0148 & 0.0071 & 2.0685 & 0.0685 & 0.3199 \\
\hline
\end{tabular}


Table 3. The simulated means, biases and MSE's for the estimators of the parameters $a, b=0.5$ and $\theta=2$.

\begin{tabular}{|c|c|c|c|c|c|c|c|c|c|c|}
\hline \multirow[b]{2}{*}{$a$} & \multirow{2}{*}{$n$} & \multicolumn{3}{|c|}{$\hat{a}$} & \multicolumn{3}{|c|}{$\hat{b}$} & \multicolumn{3}{|c|}{$\hat{\theta}$} \\
\hline & & Mean & Bias & MSE & Mean & Bias & MSE & Mean & Bias & MSE \\
\hline \multirow{3}{*}{0.95} & 30 & 0.9432 & -0.0068 & 0.0008 & 0.5826 & 0.0823 & 0.0471 & 2.1405 & 0.1405 & 0.7726 \\
\hline & 50 & 0.9473 & -0.0027 & 0.0002 & 0.5280 & 0.0280 & 0.0201 & 2.0474 & 0.0474 & 0.3559 \\
\hline & 100 & 0.9488 & -0.0012 & 0.0000 & 0.5168 & 0.0168 & 0.0070 & 2.0437 & 0.0437 & 0.1769 \\
\hline \multirow{3}{*}{0.99} & 30 & 0.9870 & -0.0030 & 0.0005 & 0.5714 & 0.0714 & 0.0485 & 2.1306 & 0.1306 & 0.7265 \\
\hline & 50 & 0.9887 & -0.0013 & 0.0001 & 0.5434 & 0.0434 & 0.0217 & 2.0685 & 0.0685 & 0.4315 \\
\hline & 100 & 0.9897 & -0.0003 & 0.0000 & 0.5164 & 0.0164 & 0.0069 & 2.0462 & 0.0462 & 0.1818 \\
\hline \multirow{3}{*}{1.01} & 30 & 1.0094 & -0.0006 & 0.0006 & 0.5797 & 0.0797 & 0.0485 & 2.1995 & 0.1995 & 1.0030 \\
\hline & 50 & 1.0098 & -0.0002 & 0.0001 & 0.5417 & 0.0417 & 0.0205 & 2.0100 & 0.0100 & 0.4552 \\
\hline & 100 & 1.0101 & 0.0001 & 0.0000 & 0.5167 & 0.0167 & 0.0067 & 2.0091 & 0.0091 & 0.1939 \\
\hline \multirow{3}{*}{1.05} & 30 & 1.0538 & 0.0038 & 0.0007 & 0.5665 & 0.0665 & 0.0460 & 2.2196 & 0.2196 & 0.9326 \\
\hline & 50 & 1.0517 & 0.0017 & 0.0002 & 0.5422 & 0.0422 & 0.0210 & 2.0776 & 0.0776 & 0.4333 \\
\hline & 100 & 1.0514 & 0.0014 & 0.0000 & 0.5215 & 0.0215 & 0.0073 & 2.0734 & 0.0734 & 0.1943 \\
\hline
\end{tabular}

Table 4. The simulated means, biases and MSE's for the estimators of the parameters $a, b=-0.5$ and $\theta=2$.

\begin{tabular}{|c|c|c|c|c|c|c|c|c|c|c|}
\hline \multirow{2}{*}{$a$} & \multirow[b]{2}{*}{$n$} & \multicolumn{3}{|c|}{$\hat{a}$} & \multicolumn{3}{|c|}{$\hat{b}$} & \multicolumn{3}{|c|}{$\theta$} \\
\hline & & Mean & Bias & MSE & Mean & Bias & MSE & Mean & Bias & MSE \\
\hline \multirow{3}{*}{0.95} & 30 & 0.9442 & -0.0058 & 0.0007 & -0.4244 & 0.0755 & 0.0463 & 2.1501 & 0.1501 & 0.7646 \\
\hline & 50 & 0.9468 & -0.0032 & 0.0002 & -0.4564 & 0.0436 & 0.0191 & 2.0804 & 0.0804 & 0.3902 \\
\hline & 100 & 0.9484 & -0.0016 & 0.0000 & -0.4808 & 0.0192 & 0.0072 & 2.0284 & 0.0284 & 0.1943 \\
\hline \multirow{3}{*}{0.99} & 30 & 0.9878 & -0.0022 & 0.0006 & -0.4280 & 0.0720 & 0.0444 & 2.1792 & 0.1792 & 0.8176 \\
\hline & 50 & 0.9895 & -0.0005 & 0.0001 & -0.4632 & 0.0368 & 0.0211 & 2.1135 & 0.1134 & 0.4164 \\
\hline & 100 & 0.9898 & -0.0002 & 0.0000 & -0.4871 & 0.0129 & 0.0074 & 2.0546 & 0.0546 & 0.1969 \\
\hline \multirow{3}{*}{1.01} & 30 & 1.0083 & -0.0017 & 0.0006 & -0.4247 & 0.0753 & 0.0496 & 2.1479 & 0.1479 & 0.7872 \\
\hline & 50 & 1.0099 & -0.0001 & 0.0001 & -0.4562 & 0.0438 & 0.0199 & 2.1279 & 0.1279 & 0.4711 \\
\hline & 100 & 1.0101 & 0.0001 & 0.0000 & -0.4875 & 0.0125 & 0.0067 & 2.0363 & 0.0363 & 0.1933 \\
\hline \multirow{3}{*}{1.05} & 30 & 1.0530 & 0.0012 & 0.0007 & -0.4256 & 0.0744 & 0.0453 & 2.1644 & 0.1644 & 0.9015 \\
\hline & 50 & 1.0522 & 0.0010 & 0.0002 & -0.4576 & 0.0424 & 0.0196 & 2.1459 & 0.1459 & 0.4606 \\
\hline & 100 & 1.0509 & 0.0009 & 0.0000 & -0.4825 & 0.0175 & 0.0073 & 2.0505 & 0.0505 & 0.2076 \\
\hline
\end{tabular}

Table 5. The simulated means, biases and MSE's for the estimators of the parameters $a, b=2$ and $\theta=0.5$.

\begin{tabular}{|c|c|c|c|c|c|c|c|c|c|c|}
\hline \multirow[b]{2}{*}{$a$} & \multirow[b]{2}{*}{$n$} & \multicolumn{3}{|c|}{$\hat{a}$} & \multicolumn{3}{|c|}{$\hat{b}$} & \multicolumn{3}{|c|}{$\hat{\theta}$} \\
\hline & & Mean & Bias & MSE & Mean & Bias & MSE & Mean & Bias & MSE \\
\hline \multirow{3}{*}{0.95} & 30 & 0.9473 & -0.0027 & 0.0005 & 2.0777 & 0.0777 & 0.0457 & 0.5126 & 0.0126 & 0.0378 \\
\hline & 50 & 0.9470 & -0.0030 & 0.0002 & 2.0478 & 0.0478 & 0.0207 & 0.4951 & -0.0049 & 0.0210 \\
\hline & 100 & 0.9491 & -0.0009 & 0.0000 & 2.0145 & 0.0145 & 0.0071 & 0.5016 & 0.0016 & 0.0111 \\
\hline \multirow{3}{*}{0.99} & 30 & 0.9912 & 0.0012 & 0.0005 & 2.0841 & 0.0841 & 0.0486 & 0.5161 & 0.0161 & 0.0377 \\
\hline & 50 & 0.9894 & -0.0006 & 0.0001 & 2.0431 & 0.0431 & 0.0197 & 0.5048 & 0.0048 & 0.0222 \\
\hline & 100 & 0.9898 & -0.0002 & 0.0000 & 2.0186 & 0.0186 & 0.0064 & 0.5004 & 0.0004 & 0.0109 \\
\hline \multirow{3}{*}{1.01} & 30 & 1.0116 & 0.0016 & 0.0006 & 2.0680 & 0.0680 & 0.0464 & 0.5184 & 0.0184 & 0.0411 \\
\hline & 50 & 1.0111 & 0.0011 & 0.0001 & 2.0441 & 0.0441 & 0.0203 & 0.5109 & 0.0109 & 0.0239 \\
\hline & 100 & 1.0102 & 0.0002 & 0.0000 & 2.0141 & 0.0141 & 0.0070 & 0.5019 & 0.0019 & 0.0102 \\
\hline \multirow{3}{*}{1.05} & 30 & 1.0563 & 0.0063 & 0.0008 & 2.0739 & 0.0739 & 0.0486 & 0.5140 & 0.0140 & 0.0389 \\
\hline & 50 & 1.0541 & 0.0041 & 0.0002 & 2.0454 & 0.0454 & 0.0201 & 0.5180 & 0.0180 & 0.0209 \\
\hline & 100 & 1.0513 & 0.0013 & 0.0001 & 2.0173 & 0.0173 & 0.0073 & 0.5059 & 0.0059 & 0.0105 \\
\hline
\end{tabular}


Table 6. The simulated means, biases and MSE's for the estimators of the parameters $a, b=-2$ and $\theta=0.5$.

\begin{tabular}{|c|c|c|c|c|c|c|c|c|c|c|}
\hline \multirow{2}{*}{$\boldsymbol{a}$} & $\boldsymbol{n}$ & \multicolumn{3}{|c|}{$\hat{\boldsymbol{a}}$} & \multicolumn{3}{|c|}{$\hat{\boldsymbol{b}}$} & \multicolumn{3}{|c|}{$\widehat{\boldsymbol{\theta}}$} \\
\cline { 2 - 11 } & & Mean & Bias & MSE & Mean & Bias & MSE & Mean & Bias & MSE \\
\hline \multirow{3}{*}{$\mathbf{0 . 9 5}$} & $\mathbf{3 0}$ & 0.9481 & -0.0019 & 0.0005 & -1.9341 & 0.0659 & 0.0418 & 0.5114 & 0.0114 & 0.0326 \\
\cline { 2 - 11 } & $\mathbf{5 0}$ & 0.9477 & -0.0023 & 0.0001 & -1.9562 & 0.0438 & 0.0210 & 0.5024 & 0.0024 & 0.0226 \\
\cline { 2 - 11 } & $\mathbf{1 0 0}$ & 0.9488 & -0.0012 & 0.0000 & -1.9814 & 0.0186 & 0.0070 & 0.4991 & -0.0009 & 0.0102 \\
\hline \multirow{3}{*}{$\mathbf{0 . 9 9}$} & $\mathbf{3 0}$ & 0.9914 & 0.0014 & 0.0005 & -1.9258 & 0.0742 & 0.0455 & 0.5142 & 0.0142 & 0.0327 \\
\cline { 2 - 11 } & $\mathbf{5 0}$ & 0.9898 & -0.0002 & 0.0001 & -1.9672 & 0.0328 & 0.0195 & 0.5097 & 0.0097 & 0.0242 \\
\cline { 2 - 11 } & $\mathbf{1 0 0}$ & 0.9900 & 0.0000 & 0.0000 & -1.9843 & 0.0157 & 0.0068 & 0.4998 & -0.0002 & 0.0098 \\
\hline \multirow{3}{*}{$\mathbf{1 . 0 1}$} & $\mathbf{3 0}$ & 1.0130 & 0.0030 & 0.0005 & -1.9270 & 0.0730 & 0.0486 & 0.5208 & 0.0208 & 0.0396 \\
\cline { 2 - 10 } & $\mathbf{5 0}$ & 1.0109 & 0.0009 & 0.0001 & -1.9525 & 0.0476 & 0.0201 & 0.5036 & 0.0036 & 0.0216 \\
\cline { 2 - 10 } & $\mathbf{1 0 0}$ & 1.0105 & 0.0005 & 0.0000 & -1.9814 & 0.0186 & 0.0072 & 0.5023 & 0.0023 & 0.0099 \\
\hline \multirow{3}{*}{$\mathbf{1 . 0 5}$} & $\mathbf{3 0}$ & 1.0543 & 0.0043 & 0.0008 & -1.9322 & 0.0678 & 0.0423 & 0.5094 & 0.0094 & 0.0366 \\
\cline { 2 - 10 } & $\mathbf{5 0}$ & 1.0529 & 0.0029 & 0.0002 & -1.9608 & 0.0392 & 0.0201 & 0.5104 & 0.0104 & 0.0223 \\
\cline { 2 - 10 } & $\mathbf{1 0 0}$ & 1.0515 & 0.0015 & 0.0000 & -1.9816 & 0.0183 & 0.0078 & 0.5108 & 0.0108 & 0.0120 \\
\hline
\end{tabular}

Table 7. The simulated means, biases and MSE's for the estimators of the parameters $a, b=0.5$ and $\theta=0.5$.

\begin{tabular}{|c|c|c|c|c|c|c|c|c|c|c|}
\hline \multirow{2}{*}{$\boldsymbol{a}$} & $\boldsymbol{n}$ & \multicolumn{3}{|c|}{$\hat{\boldsymbol{a}}$} & \multicolumn{3}{c|}{$\hat{\boldsymbol{b}}$} & \multicolumn{3}{c|}{$\widehat{\boldsymbol{\theta}}$} \\
\cline { 2 - 11 } & & Mean & Bias & MSE & Mean & Bias & MSE & Mean & Bias & MSE \\
\hline \multirow{3}{*}{$\mathbf{0 . 9 5}$} & $\mathbf{3 0}$ & 0.9466 & -0.0034 & 0.0006 & 0.5753 & 0.0753 & 0.0452 & 0.5072 & 0.0072 & 0.0357 \\
\cline { 2 - 11 } & $\mathbf{5 0}$ & 0.9478 & -0.0022 & 0.0001 & 0.5421 & 0.0421 & 0.0205 & 0.5057 & 0.0057 & 0.0216 \\
\cline { 2 - 11 } & $\mathbf{1 0 0}$ & 0.9492 & -0.0008 & 0.0000 & 0.5161 & 0.0161 & 0.0073 & 0.5026 & 0.0026 & 0.0102 \\
\hline \multirow{3}{*}{$\mathbf{0 . 9 9}$} & $\mathbf{3 0}$ & 0.9895 & -0.0005 & 0.0005 & 0.5811 & 0.0811 & 0.0518 & 0.5022 & 0.0022 & 0.0363 \\
\cline { 2 - 11 } & $\mathbf{5 0}$ & 0.9899 & -0.0000 & 0.0001 & 0.5470 & 0.0470 & 0.0200 & 0.5083 & 0.0083 & 0.0220 \\
\cline { 2 - 11 } & $\mathbf{1 0 0}$ & 0.9899 & -0.0000 & 0.0000 & 0.5240 & 0.0240 & 0.0074 & 0.5041 & 0.0041 & 0.0094 \\
\hline \multirow{3}{*}{$\mathbf{1 . 0 1}$} & $\mathbf{3 0}$ & 1.0124 & 0.0024 & 0.0007 & 0.5771 & 0.0771 & 0.0496 & 0.5127 & 0.0127 & 0.0419 \\
\cline { 2 - 10 } & $\mathbf{5 0}$ & 1.0109 & 0.0009 & 0.0001 & 0.5383 & 0.0383 & 0.0199 & 0.5083 & 0.0083 & 0.0205 \\
\cline { 2 - 10 } & $\mathbf{1 0 0}$ & 1.0100 & 0.0000 & 0.0000 & 0.5148 & 0.0148 & 0.0069 & 0.5011 & 0.0011 & 0.0105 \\
\hline \multirow{3}{*}{$\mathbf{1 . 0 5}$} & $\mathbf{3 0}$ & 1.0567 & 0.0067 & 0.0009 & 0.5789 & 0.0789 & 0.0507 & 0.5152 & 0.0152 & 0.0390 \\
\cline { 2 - 10 } & $\mathbf{5 0}$ & 1.0536 & 0.0036 & 0.0002 & 0.5471 & 0.0471 & 0.0215 & 0.5101 & 0.0101 & 0.0212 \\
\cline { 2 - 10 } & $\mathbf{1 0 0}$ & 1.0515 & 0.0015 & 0.0000 & 0.5181 & 0.0181 & 0.0072 & 0.5094 & 0.0094 & 0.0099 \\
\hline
\end{tabular}

Table 8. The simulated means, biases and MSE's for the estimators of the parameters $a, b=-0.5$ and $\theta=0.5$.

\begin{tabular}{|c|c|c|c|c|c|c|c|c|c|c|}
\hline \multirow{2}{*}{$a$} & \multirow{2}{*}{$n$} & \multicolumn{3}{|c|}{$\hat{a}$} & \multicolumn{3}{|c|}{$\hat{b}$} & \multicolumn{3}{|c|}{$\hat{\theta}$} \\
\hline & & Mean & Bias & MSE & Mean & Bias & MSE & Mean & Bias & MSE \\
\hline \multirow{3}{*}{0.95} & 30 & 0.9462 & -0.0038 & 0.0006 & -0.4263 & 0.0737 & 0.0477 & 0.5102 & 0.0102 & 0.0338 \\
\hline & 50 & 0.9479 & -0.0021 & 0.0002 & -0.4620 & 0.0380 & 0.0204 & 0.5020 & 0.0020 & 0.0233 \\
\hline & 100 & 0.9492 & -0.0008 & 0.0000 & -0.4870 & 0.0130 & 0.0075 & 0.4987 & -0.0013 & 0.0103 \\
\hline \multirow{3}{*}{0.99} & 30 & 0.9906 & 0.0006 & 0.0005 & -0.4322 & 0.0678 & 0.0435 & 0.5137 & 0.0137 & 0.0410 \\
\hline & 50 & 0.9902 & 0.0002 & 0.0001 & -0.4625 & 0.0375 & 0.0110 & 0.5090 & 0.0090 & 0.0214 \\
\hline & 100 & 0.9899 & -0.0001 & 0.0000 & -0.4823 & 0.0177 & 0.0077 & 0.5021 & 0.0021 & 0.0111 \\
\hline \multirow{3}{*}{1.01} & 30 & 1.0126 & 0.0026 & 0.0006 & -0.4113 & 0.0887 & 0.0485 & 0.5136 & 0.0136 & 0.0392 \\
\hline & 50 & 1.0109 & 0.0009 & 0.0001 & -0.4665 & 0.0335 & 0.0184 & 0.5083 & 0.0083 & 0.0225 \\
\hline & 100 & 1.0103 & 0.0003 & 0.0000 & -0.4822 & 0.0178 & 0.0078 & 0.5019 & 0.0019 & 0.0104 \\
\hline \multirow{3}{*}{1.05} & 30 & 1.0564 & 0.0064 & 0.0008 & -0.4264 & 0.0736 & 0.0447 & 0.5213 & 0.0213 & 0.0405 \\
\hline & 50 & 1.0528 & 0.0028 & 0.0002 & -0.4623 & 0.0377 & 0.0199 & 0.5042 & 0.0042 & 0.0210 \\
\hline & 100 & 1.0513 & 0.0013 & 0.0000 & -0.4845 & 0.0155 & 0.0073 & 0.5086 & 0.0086 & 0.0111 \\
\hline
\end{tabular}


By the help of the asymptotic joint distribution of the estimators, the marginal asymptotic distribution of each estimator is obtained, and with marginal distributions, the statistical properties such as asymptotic unbiasedness, consistency and efficiency can be investigated. However, convergence rates of the asymptotic properties for these estimators are not explicit. For this reason, it is of importance to evaluate the small sample performance of the proposed estimators by a simulation study.

According to the simulation results given in Tables 1-8, although the performance of the ML estimators of the parameters $a$ and $b$ is well even for the sample size $n=30$, this comment cannot be made for the performance of estimator of $\theta$. It seems that the converges rate for estimator of $\theta$ is slower than the others. However, it can be easily seen that both the bias and MSE values become smaller as $n$ increases for all the estimators of the parameters.

It is well known that the diagonal elements of $I^{-1}$, that is $u_{11}, u_{22}$, and $u_{33}$, are the minimum variance bounds (MVBs) for the parameters $a, b$ and $\theta$. The simulated variances of the ML estimators and corresponding MVB values for $a=0.95, b=2$, and $\theta=2$ are given in the following Table 9 .

Table 9. The simulated variances and corresponding MVBs values.

\begin{tabular}{|c|c|c|c|c|c|c|}
\hline \multirow{2}{*}{$\boldsymbol{n}$} & \multicolumn{2}{|c|}{ Simulated Variance } & \multicolumn{3}{|c|}{ MVBs } \\
\cline { 2 - 7 } & $\hat{\boldsymbol{a}}$ & $\hat{\boldsymbol{b}}$ & $\hat{\boldsymbol{\theta}}$ & $\hat{\boldsymbol{a}}$ & $\hat{\boldsymbol{b}}$ & $\hat{\boldsymbol{\theta}}$ \\
\hline $\mathbf{3 0}$ & 0.00064 & 0.04121 & 0.64175 & 0.00057 & 0.03639 & 0.52812 \\
$\mathbf{5 0}$ & 0.00018 & 0.01859 & 0.40265 & 0.00015 & 0.01734 & 0.32417 \\
$\mathbf{1 0 0}$ & 0.00004 & 0.00708 & 0.16717 & 0.00004 & 0.00671 & 0.16372 \\
\hline
\end{tabular}

As can be seen from the Table 9 that the simulated variances of the ML estimators and corresponding MVB values are getting closer as $n$ increases. According to the simulation study, it can be concluded that the proposed estimators are highly efficient estimators for the parameters $a, b$ and $\theta$.

\section{Applications}

In this section, we consider three real world data sets given in $[1,6,10]$, namely the hydraulic system failure data (Data set 1), USS Halfbeak No. 3 propulsion diesel engine failure data (Data set 2), and the Aircraft 3 data (Data set 3), respectively. Nonhomogeneous Poisson process with power law intensity is considered as the model for the Data set 1 by $[6,7]$ shows that the GP is the suitable model for the Data set 2 . However, $\mathrm{Wu}$ [12] applies the DGP to model Data set 1 and Data set 2 and shows that the DGP outperforms the other models for these data sets in terms of the root mean square errors according to the least square method. Since Wu [12] finds the DGP as the suitable model on Data set 1 and Data set 2, we do not consider model comparisons for these data sets in this paper. However, for the Data set 3, which is known to be consistent with GP model [7], it is required to show that the DGP performs better than the GP model. For this purpose, we consider $\log \mathrm{L}$ and AIC as comparison criteria to determine the suitable model. Moreover, to apply the estimation procedure developed in this study, we need to show that the data sets come from the DGP with particular exponential distribution. We propose following approach to see this result. First, we assume that the data sets follow a DGP with particular exponential distribution. Then, we obtain the ML estimators of the parameters $a, b$, and $\theta$ for each data set. If we can show that the predictions $\hat{y}_{k}$ 's are iid exponential random variables then, the DGP with particular exponential distribution can be used for modeling of the data set. For this purpose, the predictions $\hat{y}_{k}$ 's are obtained from $\hat{a}^{k-1} x_{k}^{(1+\log (k))^{\hat{b}}}, k=1,2, \ldots, n$ where $\hat{a}$ and $\hat{b}$ are the ML estimators of the parameters $a$ and $b$. The Kolmogorov-Smirnov (KS) test is applied to measure the goodness 
of fitness of the predictions for exponential distribution with mean $\hat{\theta}$. By assuming the Data set 1 and Data set 2 follow a DGP with particular exponential distribution, the ML estimators of the parameters $a, b$, and $\theta$, the $\mathrm{KS}$ statistic and corresponding $p$-value are presented in the following Table 10 for these data sets.

Table 10. ML Estimators, KS Test Statistic and $p$-value of the data sets.

\begin{tabular}{|l|c|c|c|c|c|}
\hline & \multicolumn{3}{|c|}{ ML Estimators } & & \\
\cline { 2 - 6 } Data set 1 & $\hat{\boldsymbol{a}}$ & $\hat{\boldsymbol{b}}$ & $\hat{\boldsymbol{\theta}}$ & KS Test Statistic & $p$-value \\
\cline { 2 - 6 } Data set 2 & 1.031119 & 0.416095 & 301.068 & 0.1288 & 0.7543 \\
Data & -0.109328 & 574.827 & 0.1193 & 0.2439 \\
\hline
\end{tabular}

As seen from Table 10 that the DGP with exponential distribution can be used as model for the Data set 1 and Data set 2. Thus, estimates of the parameters can be taken as given in Table 10 above.

Now, consider Data set 3. Let us calculate the ML estimators of the parameters $a, b$, and $\theta$ for both DGP and GP models for this data set. By using these estimates of the parameters, the values of $\log \mathrm{L}$ and AIC are also calculated and presented in the following Table 11 together with the estimates of the parameters.

Table 11. Parameter Estimates and LogL and AIC values for DGP and GP models.

\begin{tabular}{|c|c|c|c|c|}
\hline & \multicolumn{2}{|c|}{ DGP } & \multicolumn{2}{c|}{ GP } \\
\hline LogL & \multicolumn{2}{|c|}{-153.913} & \multicolumn{1}{c|}{-156.349} \\
AIC & \multicolumn{2}{|c|}{313.826} & \multicolumn{2}{c|}{316.697} \\
\hline \multirow{3}{*}{ Estimates } & $\hat{\boldsymbol{b}}$ & 0.4122 & & \\
& $\hat{\boldsymbol{a}}$ & 0.9108 & $\hat{\boldsymbol{a}}$ & 0.9709 \\
& $\hat{\boldsymbol{\theta}}$ & 111.905 & $\hat{\boldsymbol{\theta}}$ & 53.381 \\
\hline
\end{tabular}

It can be concluded from Table 11 that the DGP model is better than the GP model according to the criteria $\log \mathrm{L}$ and AIC. After obtaining this result, we show that Data set 3 follows a DGP with a particular exponential distribution. For this purpose, the KS statistic and corresponding $p$ value are presented in the following Table 12 for this data set.

Table 12. KS Test Statistic and $p$-value of the Data set 3.

\begin{tabular}{|l|c|c|}
\hline & KS Test Statistic & $p$-value \\
\hline Data set 3 & 0.0949 & 0.9342 \\
\hline
\end{tabular}

As can be seen from Table 12, the DGP with exponential distribution can be used as model for the Data set 3. Thus, estimates of the parameters can be taken as given in Table 11 above.

\section{Conclusions}

In this study, statistical inference problem of the DGP which is a novel model in stochastic processes is studied when the distribution of the first interarrival time has an exponential distribution. The estimators of the parameters $a, b$, and $\theta$ are obtained by using the ML method. Then, we investigate the statistical properties of the ML estimators. By carrying out an extensive simulation study, the performance of the estimators is evaluated. It is shown that three real world applications can be fitted by the DGP model with a particular exponential distribution. For these data sets, the estimates of the parameters are given. 


\section{References}

[1] H. Ascher and H. Feingold, Repairable Systems Reliability: Modelling, Inference, Misconceptions and Their Causes, Marcel Dekker, 1984.

[2] J. Bai and H. Pham, Repair-limit risk-free warranty policies with imperfect repair, IEEE Trans. Syst., Man, Cybern. A, Syst. Humans 36 (6), 756-772, 2005.

[3] O.E. Barndorff-Nielsen and D.R. Cox, Inference and Asymptotics, Chapman and Hall, 1994.

[4] J.S. Chan, P.L. Yu, Y. Lam and A.P. Ho, Modelling SARS data using threshold geometric process, Stat. Med. 25 (11), 1826-1839, 2006.

[5] J.S. Chan, S.B. Choy and C.P. Lam, Modeling electricity price using a threshold conditional autoregressive geometric process jump model, Comm. Statist. Theory Methods 43 (1012), 2505-2515, 2014.

[6] U. Kumar and B. Klefsjö, Reliability analysis of hydraulic systems of LHD machines using the power law process model, Reliab. Eng. Syst. Saf. 35 (3), 217-224, 1992.

[7] Y. Lam, The Geometric Process and Its Applications, Word Scientific, 2007.

[8] M. Park and H. Pham, Warranty cost analyses using quasi-renewal processes for multicomponent systems, IEEE Trans. Syst., Man, Cybern. A, Syst. Human 40 (6), 1329-1340, 2010.

[9] H. Pham and H. Wang, A quasi-renewal process for software reliability and testing costs, IEEE Trans. Syst., Man, Cybern. A, Syst. Human 31 (6), 623-631, 2001.

[10] F. Proschan, Theoretical explanation of observed decreasing failure rate, Technometrics 42 (1), 7-11, 2000.

[11] H. Wang and H. Pham, A quasi-renewal process and its applications in imperfect maintenance, Internat. J. Systems Sci. 27 (10), 1055-1062, 1996.

[12] S. Wu, Doubly geometric process and applications, J. Oper. Res. Soc. 69 (1), 66-67, 2018.

[13] S. Wu and P. Scarf, Decline and repair, and covariate effects, European J. Oper. Res. 244 (1), 219-226, 2015.

[14] S. Wu and G. Wang, The semi-geometric process and some properties, IMA J. Manag. Math. 29 (2), 229-245, 2018.

[15] M. Zhang, M. Xie and O. Gaudoin, A bivariate maintenance policy for multi-state repairable systems with monotone process, IEEE Trans. Rel. 62 (4), 876-886, 2013.

\section{Appendix}

The derivation of $\boldsymbol{I}^{-\mathbf{1}}$. The second derivations of the log-likelihood function given in with respect Equation 2.1 to the parameters $a, b$, and $\theta$ are obtained as

$$
\begin{aligned}
\frac{\partial^{2} L n L}{\partial a^{2}}= & -\frac{n(n-1)}{2 a^{2}}-\frac{1}{\theta}\left(\sum_{k=1}^{n}(k-1)(k-2) a^{k-3} x_{k}^{(1+\log (k))^{b}}\right) \\
\frac{\partial^{2} L n L}{\partial a \partial b}= & -\frac{1}{\theta}\left(\sum_{k=1}^{n}(k-1) a^{k-2} x_{k}^{(1+\log (k))^{b}} \ln \left(x_{k}\right)(1+\log (k))^{b} \ln (1+\log (k))\right) \\
\frac{\partial^{2} L n L}{\partial a \partial \theta}= & \frac{1}{\theta^{2}}\left(\sum_{k=1}^{n}(k-1) a^{k-2} x_{k}^{(1+\log (k))^{b}}\right) \\
\frac{\partial^{2} L n L}{\partial b^{2}}= & \left(-\frac{1}{\theta}\left(\sum_{k=1}^{n} a^{k-1} \ln \left(x_{k}\right)(1+\log (k))^{b}(\ln (1+\log (k)))^{2} x_{k}^{(1+\log (k))^{b}}\right.\right. \\
& \left.\left.\left(1+(1+\log (k))^{b} \ln \left(x_{k}\right)\right)\right)\right)+\left(\sum_{k=1}^{n} \ln \left(x_{k}\right) \ln (1+\log (k))\right)^{2}(1+\log (k))^{b}
\end{aligned}
$$




$$
\begin{aligned}
& \frac{\partial^{2} \operatorname{LnL}}{\partial b \partial \theta}=\frac{1}{\theta^{2}}\left(\sum_{k=1}^{n} a^{k-1} x_{k}^{(1+\log (k))^{b}} \ln \left(x_{k}\right)(1+\log (k))^{b} \ln (1+\log (k))\right) \\
& \frac{\partial^{2} \operatorname{LnL}}{\partial \theta^{2}}=\frac{n}{\theta^{2}}-\frac{2}{\theta^{3}}\left(\sum_{k=1}^{n} a^{k-1} x_{k}^{(1+\log (k))^{b}}\right) .
\end{aligned}
$$

Since $Y_{k}=a^{k-1} X_{k}^{(1+\log (k))^{b}}, k=1,2, \ldots$ and $Y_{k}$ 's are independent and exponentially distributed random variables with the same mean $\theta$, it can be easily calculated that for $k=1,2, \ldots$

$$
\begin{aligned}
& E\left(Y_{k}\right)=\theta \\
& E\left(X_{k}^{(1+\log (k))^{b}}\right)=\frac{\theta}{a^{k-1}}, \\
& E\left(\ln \left(X_{k}^{(1+\log (k))^{b}}\right)\right)=-0.577216+\ln (\theta)-\ln \left(a^{k-1}\right), \\
& E\left(X_{k}^{(1+\log (k))^{b}} \ln \left(X_{k}^{(1+\log (k))^{b}}\right)\right)=\frac{\theta}{a^{k-1}}\left(0.422784+\ln (\theta)-\ln \left(a^{k-1}\right)\right)
\end{aligned}
$$

and

$$
\begin{aligned}
E\left(X_{k}^{(1+\log (k))^{b}}\left(\ln \left(X_{k}^{(1+\log (k))^{b}}\right)\right)^{2}\right)= & \frac{1}{a^{k-1}}((0.16667 \theta(4.94208+6 \ln (\theta)(0.845569+\ln (\theta)))) \\
& \left.-2 \ln \left(a^{k-1}\right)(\theta(0.422784+\ln (\theta)))+\left(\ln \left(a^{k-1}\right)\right)^{2} \theta\right) .
\end{aligned}
$$

Then, the expected values of the second derivatives are

$$
\begin{aligned}
E\left(-\frac{\partial^{2} L n L}{\partial a^{2}}\right)= & \frac{2 n^{3}-3 n^{2}+n}{6 a^{2}}, \\
E\left(-\frac{\partial^{2} L n L}{\partial a \partial b}\right)= & \frac{1}{a}\left(\sum_{k=1}^{n}(k-1) \ln (1+\log (k))\left(0.422784+\ln (\theta)-\ln \left(a^{k-1}\right)\right)\right), \\
E\left(-\frac{\partial^{2} L n L}{\partial a \partial \theta}\right)= & \frac{n-n^{2}}{2 a \theta}, \\
E\left(-\frac{\partial^{2} L n L}{\partial b^{2}}\right)= & \sum_{k=1}^{n}(\ln (1+\log (k)))^{2}(1+((0.16667(4.94208+6 \ln (\theta)(0.845569+\ln (\theta)))) \\
& \left.\left.-2 \ln \left(a^{k-1}\right)(0.422784+\ln (\theta))+\left(\ln \left(a^{k-1}\right)\right)^{2}\right)\right), \\
E\left(-\frac{\partial^{2} L n L}{\partial b \partial \theta}\right)= & -\frac{1}{\theta}\left(\sum_{k=1}^{n} \ln (1+\log (k))\left(0.422784+\ln (\theta)-\ln \left(a^{k-1}\right)\right)\right), \\
\text { and } & \\
E\left(-\frac{\partial^{2} L n L}{\partial \theta^{2}}\right)= & \frac{n}{\theta^{2}} .
\end{aligned}
$$

Thus, the elements of the Fisher information matrix $I$ are obtained. The inverse of this matrix is given in Equation 2.3. 